\title{
Emergency Treatment and Nursing Care of a Patient with Multiple Injuries and Right Main Bronchus Rupture
}

\author{
Tianran Li' ${ }^{1}$, Lanping Shi ${ }^{2}$ \\ ${ }^{1}$ Changjiang University, Jingzhou, China \\ ${ }^{2}$ The University of Hong Kong-Shenzhen Hospital, Shenzhen, China \\ Email:2414220761@qq.com
}

How to cite this paper: Li, T.R. and Shi, L.P. (2021) Emergency Treatment and Nursing Care of a Patient with Multiple Injuries and Right Main Bronchus Rupture. Journal of Biosciences and Medicines, 9, 231-245. https://doi.org/10.4236/jbm.2021.97021

Received: June 16, 2021

Accepted: July 26, 2021

Published: July 29, 2021

Copyright () 2021 by author(s) and Scientific Research Publishing Inc. This work is licensed under the Creative Commons Attribution International License (CC BY 4.0).

http://creativecommons.org/licenses/by/4.0/

\begin{abstract}
Objective: To investigate the rescue and nursing process of a trauma patient with multiple injuries and rupture of the right main bronchus. Methods: A patient with multiple injuries and rupture of the right main bronchus admitted to the emergency department of the Shenzhen Hospital of the University of Hong Kong was selected as the research object on December 11, 2019. Results: In this case, the medical team treated the patient under the guidance of ATLS (Advanced Traumatic Life Support), and for the first time in our department, we used bronchoscopy to replace the double-lumen endotracheal tube for left lung single-lung ventilation, finally the patient was successfully treated. Conclusion: Through a literature search, it is found that the main bronchus rupture is less common in clinical practice. In the trauma group, the use of fiberoptic bronchoscopy and the replacement of a double-lumen tracheal tube for left lung single-lung ventilation can improve the treatment rate of such patients and is worthy of clinical application.
\end{abstract}

\section{Keywords}

Multiple Injuries, Right Main Bronchus, Bronchial Rupture, Nursing,

Treatment

\section{Introduction}

Severe blunt chest trauma can cause rupture or rupture of the trachea and bronchus [1], the incidence of which is only $1 \%$ and the on-site mortality rate is as high as $30 \%$ [2]. Tracheal injury accounts for about $74 \%$ (left side of Figure 1 ), of which the right bronchus is the most common [1] [2], and the proportion is as high as $25 \%$ [3]. Damage to the right main bronchus is often accompanied 


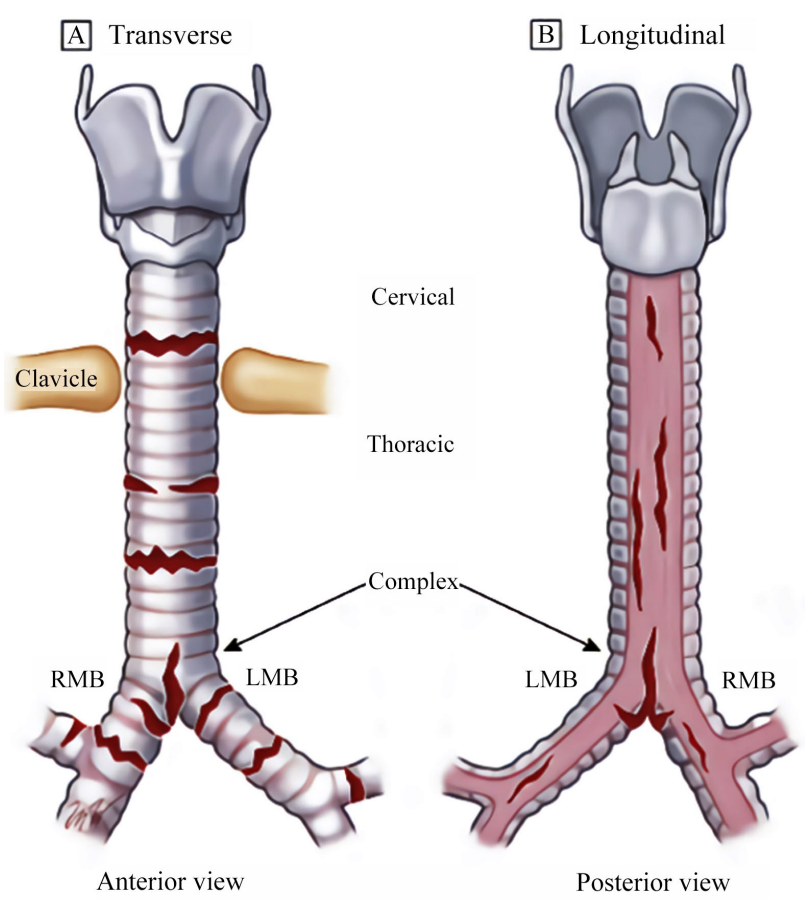

Figure 1. Schematic diagram of bronchial injury.

by damage to the lung and chest wall. It is not easy to detect them at an early stage, and diagnosis is usually difficult.

Once the right main bronchus ruptures, the air immediately enters the chest cavity and forms subcutaneous emphysema on the neck, chest, and back. The main clinical symptoms are dyspnea, hoarseness, hemoptysis, subcutaneous emphysema, and weakened or disappeared breath sounds. Because the symptoms and signs of tracheal rupture are similar to those of chest trauma, it is easy to confuse them. If the medical staff know little about this, it will be impossible to find out in time that the patient is indeed suffering from a bronchial injury [4]. Therefore, after the patient has dealt with the chest injury, the symptoms of dyspnea may still not be relieved, which delays the patient's treatment, and even leads to the death of the patient, which causes serious consequences and medical disputes [5].

ATLS is a guideline for the treatment of trauma patients formulated by the American College of Surgeons and the Trauma Committee [6]. It has a history of more than 40 years and has now been adopted by more than 60 countries in the world. However, in my country, few medical teams use ATLS as a guide for diagnosis and treatment. In this case, the medical team of our hospital used ATLS to conduct emergency treatment for tracheal rupture, and found the patient's right main bronchus injury in time. Besides, for the first time in our department, we used fiberoptic bronchoscopy to replace the double-lumen tracheal tube for left lung single-lung ventilation, so that the patient was finally successfully treated. Because this case is difficult and relatively rare in clinical practice, it has great instructive significance to medical staff. This article elaborates and discusses the rescue and nursing process of this patient, aims at sharing this case to inspire nursing staff to think about the care of rare bronchial injury patients, summarize 
the experience, and improve the level of emergency care.

\section{Case Presentation}

On December 11, 2019, a patient with multiple injuries and rupture of the right main bronchus was admitted to our hospital. Compared with traditional treatment methods, the use of fiberoptic bronchoscopy and replacement of doublelumen endotracheal tubes for patients with left lung single-lung ventilation can increase the treatment rate, shorten the hospital stay, and promote rapid recovery of patients. The report is as follows:

\subsection{Basic Patient Information}

Patient's personal information: Jin, female, 50 years old; Han nationality, from Sichuan, China; Born in Shenzhen, Guangdong on May 29, 1969; Freelance.

The patient's personal history: normal development, no history of allergies, no history of life in a different place, no history of life in a non-affected area, no history of smoking, no history of smelting, no history of life in a different place, drinking, no history of radiation exposure.

Patient's past history: Appendectomy in 2008, no history of chronic diseases such as coronary heart disease, diabetes, hypertension, no history of infectious diseases such as hepatitis, typhoid fever, tuberculosis, and no history of blood transfusion. The history of vaccination is unknown.

Family history: There is no history of infectious diseases and genetic diseases in the family.

\subsection{Patient Pre-Hospital Information}

The patient was admitted to the emergency department 10 minutes after the car accident trauma. The patient was in a coma, occipital bleeding, urinary incontinence, and no vomiting. The Pre-hospital report is shown in Table 1.

\subsection{Assessment of Injury Severity (Injury Severity Score, ISS)}

Severe trauma includes 1 point for head and neck, face, chest, abdomen, and pelvis, 1 point for limbs and pelvis, 1 point for body surface, and 42 points for ISS, which is a critical injury (ISS score, ISS $<16$ is classified as a minor injury, ISS16-24 is classified as serious injury, ISS $>25$ is classified as critical injury [7]).

Table 1. Pre-Hospital MIST report.

\begin{tabular}{ll}
\hline Project & Content \\
\hline $\begin{array}{l}\text { M (Mechanism and time of injury) } \\
\text { Injury mechanism and time }\end{array}$ & The speed of the car was unknown 10 minutes ago when it was knocked down. \\
$\begin{array}{l}\text { I (Injuries found and suspected) } \\
\text { Found and suspected injuries }\end{array}$ & Unconscious, head trauma, chest trauma, scattered bruises. \\
$\begin{array}{l}\text { S (Symptoms and signs) } \\
\text { Symptoms and signs }\end{array}$ & HR: 110 times $/ \mathrm{min}, \mathrm{RR}: 34$ times $/ \mathrm{min}, \mathrm{Bp:} 140 / 74 \mathrm{mmHg}$ \\
$\begin{array}{l}\text { T (Treatment initiated) } \\
\text { Treatment measures that have been given }\end{array}$ & GCS points, $\mathrm{SpO}_{2} 36 \%$ \\
\hline
\end{tabular}




\subsection{Auxiliary Inspection}

\subsubsection{Imaging Inspection}

1) The right nasal bone may be fractured; 2) Multiple bilateral ribs, left clavicle, scapula, upper thoracic spine have multiple spinous processes and fractures, which may be accompanied by main tracheal bifurcation breaks; The right main bronchus is ruptured and the right thoracic cavity may be accompanied by blood pneumothorax; the left main bronchus is injured, the tracheal intubation is placed in the left thoracic cavity, the left lung is ventilated normally, and the left lung has multiple exudative foci; 3) Emphysema in the mediastinum, bilateral Neck subcutaneous emphysema, bilateral thoracic drainage after operation, and no obvious abnormalities in the abdominal organs.

\subsubsection{Initial Diagnosis}

1) Right main bronchial rupture, and left main bronchial injury; 2) Right blood gas chest; 3) Multiple fractures: left collarbone fracture, left shoulder blade fracture, two side rib fractures, and third metacarpal fracture of right hand.

\subsection{Rescue Process}

Table 2 shows the detailed process of rescue of patients with multiple injury right main bronchus rupture.

Table 2. Patient's rescue process.

\section{Summary of treatment}

11:28 Open the airway to the patient, place an oropharyngeal vent tube in the oropharynx, and give oxygen to the high-flow mask. Establish 2 venous accesses for the patient, and then perform blood test.

11:38 The patient was given balloon-assisted ventilation; $20 \mathrm{mg}$ etomidate and $100 \mathrm{mg}$ succinylcholine chloride were given intravenously.

11:50 The patient was successfully placed in a 7.0\# tracheal tube for sputum suction. The tracheal tube is connected to the ventilator for assisted breathing; the ventilator is set to SIMV mode, with a tidal volume of $400 \mathrm{ml}$, a respiratory rate of $14 \mathrm{times} / \mathrm{min}$, and a positive end-breathing pressure of $4 \mathrm{cmH}_{2} \mathrm{O}$.

11:54 The DR chest radiograph was performed at the bedside, and the image results are shown in Figure 2.

11:54 The patient developed ventilator resistance and was given an intravenous bolus of midazolam $2 \mathrm{mg}$ and rocuronium $30 \mathrm{mg}$.

11:58 After closed drainage of the thoracic cavity on both sides, a large number of bubbles were induced.

12:19 The patient's $\mathrm{SpO}_{2}$ fluctuates in the range of 33\% - 76\%. After bronchoscopy, it was found that his right main bronchus was damaged.

12:36 Because the patient's heart rate dropped to 41 beats/min, the blood pressure was undetectable, and the carotid artery pulsation was weak, so chest compressions were given.

12:37 The patient's heart rate rises to 66 beats per minute, and the carotid pulse can be palpable.

12:40 After intravenous bolus of $20 \mathrm{mg}$ rocuronium was given, bronchoscopy was performed again. After the tracheal tube is removed, the double-lumen tracheal tube is inserted through the auxiliary replacement of the bronchoscope. The left main bronchus was inserted into the front end of the catheter, and the insertion depth was $29 \mathrm{~cm}$. The upper end of the catheter is inflated to seal the right main bronchus. Adjust the ventilator parameters as: SIMV, VT400 ml, RR18 times/min. One-lung ventilation was given to the left lung.

12:43 Because the patient's heart rate dropped to 43 beats/min, the blood pressure was undetectable, and the carotid artery pulsation was weak, so chest compressions were given.

12:45 The patient's heart rate rose to 102 beats per minute.

12:55 The patient's blood oxygen saturation rose to $100 \%$, and his condition stabilized. After the patient went to the CT room for examination, he returned to the rescue room.

13:05 Chest CT examination and chest 3D reconstruction, the image result is shown in Figure 3.

13:13 Heart rate rises, blood pressure rises. He was given $20 \mathrm{mg}$ IV of rocuronium bromide and $2 \mathrm{mg}$ IV of midazolam.

13:30 The green channel leads directly to the operating room. 


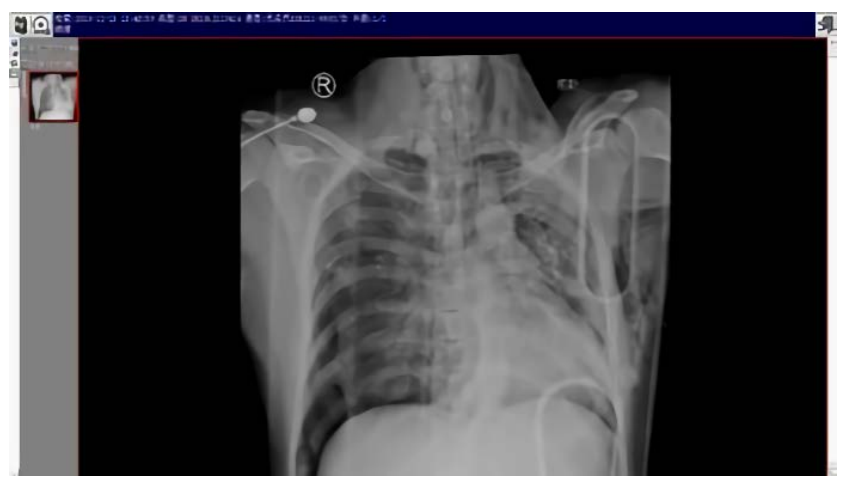

Figure 2. X-ray image of the chest at 11:48 after replacing the double cavity trachea intubation.

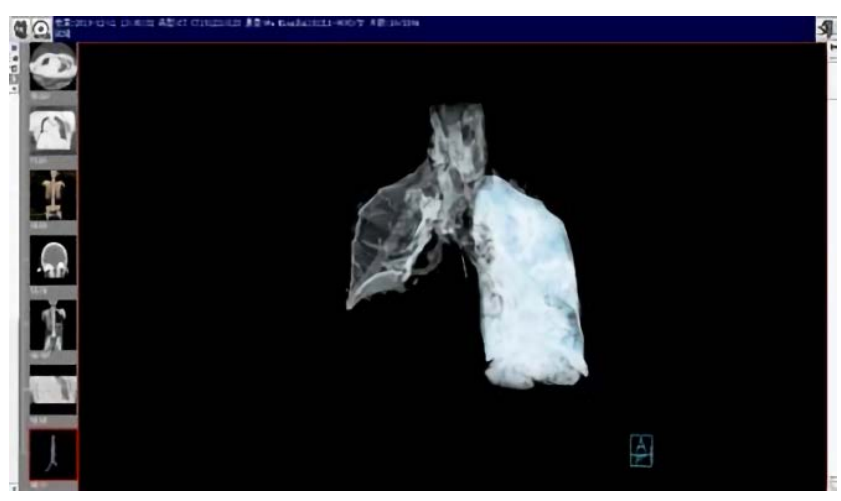

Figure 3. Chest CT examination and chest $3 \mathrm{D}$ reconstruction at 13:05.

\subsection{Surgery Information}

On December 11, 2021, the patient underwent "open reduction and internal fixation of the left clavicle under the guidance of bronchoscope", "open chest exploration", "reconstruction of semi-uplift with the aid of thoracoscopy", and "closed thoracic drainage". On December 27, 2021, the patient received "open reduction and internal fixation for the third metacarpal fracture of the right hand".

\subsection{Rescue and Outcome}

After admission to hospital, trachea intubation, ventilator-assisted breathing, chest closed drainage, pain relief, anti-infection, acid suppression, rehydration, phlegm, and other treatment. After being hospitalized in the ICU, Thoracic Surgery, Traumatology and Orthopedic Oncology, and Rehabilitation Medicine Department H, the patient was discharged on January 22, 2020.

\section{Emergency Care}

\subsection{Emergency Rating Is Rated as Class I}

Based on the patient's pre-hospital report, the trauma team is established, and the emergency team leader immediately opens the green channel and activates 
the trauma team (including Trauma Leader, Anesthesiology, ICU, Orthopedics, Neurology) upon receiving the pre-hospital first aid report.

\subsection{Preliminary Assessment}

\subsubsection{Airway Maintenance with Cervical Vertebral Protection}

Nursing assessment: 1) Patients with a small amount of nose and mouth secretions, no rhinorrhea, no otorrhea; 2) The neck brace has been fixed before arriving to the hospital, and the fixation is good; 3) Patient's jaw face is free of deformities, swelling, skull integrity, and bone rub; 4) No deformities or dislocations were found in the cervical vertebrae. The trachea is centered with no obvious airway obstruction.

Nursing diagnosis: There is a risk of suffocation.

Care goals: 1) Keep the patient's airways open; 2) Cooperate with the doctor to establish a deterministic airway; 3) No airway obstruction occurs.

Care measures: 1) Open the airway immediately, place OPA, attract oral and nasal secretions with Negative pressure, designate a person to manage the airways on the patient's head side; 2) keep cervical brace fixation, keep the spine in a straight line; 3) After the oxygen flow is adjusted to $15 \mathrm{~L} / \mathrm{min}$, the patient's SP02 has not been significantly improved. Therefore, change the balloon to assist ventilation; 4) Give intravenous bolus of etomidate $20 \mathrm{mg}$ and succinylcholine $100 \mathrm{mg}$, and follow the doctor's advice to cooperate with the doctor for tracheal intubation. After successful placement of the tube, properly fix the tracheal tube and connect the ventilator to assist breathing; 5) Patients' blood oxygen continues to be below normal, and report to the doctor; 6) Cooperate with the doctor for bedside fiberoptic bronchoscopy; 7) When replacing the tracheal tube, move quickly and gently to avoid pulling the tube; at the same time, prepare breathing balloons, syringes, tapes, and dental pads by the bed; 8) After the trachea intubation, observe diligently, clean the catheter endocrine and blood in time to avoid causing airway obstruction; 9) When attracting, the action should be gentle, and the pressure should not be too deep to avoid irritating the injured end of the trachea.

\subsubsection{Breathing and Ventilation}

Nursing assessment: 1) RR: 35 times/min, shortness of breath, purple. $\mathrm{SpO}_{2}$ : 58\% (mask oxygen $15 \mathrm{~L} / \mathrm{min}$ ); 2) Exposed patient's chest, and the bilateral thorax was symmetrical without collapse. The right thoracic undulation is worse than that on the left, the breath sounds are weakened, and the percussion is dull. Breath sounds on the left side are clear; 3) No three concave signs, no cervical veins; 4) When palpating both chest walls, there is a feeling of bony rubbing and subcutaneous emphysema in the right armpit, and heart tone is clear.

Nursing diagnosis: 1) Damaged self-breathing; 2) Damaged gas exchange, inefficient respiratory form.

Care goals: 1) Maintain oxygen saturation $\geq 94 \%$ and $\mathrm{EtCO}_{2}$ fluctuates at 35 $40 \mathrm{mmHg}$ after intubation; 2) Put in place the nursing measures for thoracic 
closed drainage; 3) Avoid ventilator lung injury to avoid aspiration caused by reflux of stomach contents.

Care measures: 1) Closely observe the patient's breathing, chest fluctuations, breathing frequency, and patterns; 2) The DR of the bedside chest shows the right blood pneumothorax. Cooperate with the doctor to perform bedside thoracic puncture catheterization and connect the thoracic cavity closed drainage bottle. Properly fix the chest tube and the closed drainage bottle of the chest cavity to avoid stretching. Observe the color and shape of the drainage fluid and the fluctuation of the fluid column. Prepare 4 toothless forceps for clamping the chest tube during transfer; 3 ) Connect the ventilator to assist breathing, then set the SIMV mode, VT $400 \mathrm{ml}$, RR 14 times/min, and PEEP $4 \mathrm{cmH}_{2} \mathrm{O}$; 4) Connect the $\mathrm{EtCO}_{2}$ monitor to observe the changes in values and waveforms; 5) Since the succinylcholine has a fast onset, the airway should be opened immediately after administration, and balloon assisted breathing or ventilator ventilation should be used to maintain the patient's oxygen supply. It is necessary to focus on the changes in the patient's heart rhythm, blood pressure and blood oxygen saturation, which may cause hypoxemia or hypotension, and even paralyze the patient's muscles, leading to respiratory and cardiac arrest and even death [7] [8]; 6) Raise the patient's bedside by 30 degrees after the doctor has confirmed that there is no abnormality in the spine; 7) Follow the doctor's advice to sedate the patient to reduce the human-machine confrontation between the patient and the ventilator; when the left lung is ventilated, closely observe the undulations of the left thorax and the subcutaneous emphysema on the right chest. If the area of subcutaneous emphysema on the right side is enlarged, it indicates that there is air leakage at the rupture of the trachea, and it needs to be reported to the doctor immediately.

\subsubsection{Circulate}

Nursing assessment: 1) HR 115 beats/min; Bp 206/106 mmHg; palpable carotid pulsation with strong pulsation; 2) A small amount of bleeding can be seen in the wound of the head and pillow, the left clavicle is swollen, the right hand is bruised and swollen with scattered abrasions, the perineum is normal, and the digital rectal examination is normal; 3) The limb end is wet and cold, and the CRT is larger than 2 seconds. There was no bulging in the abdomen, and the pelvic compression test was negative.

Nursing diagnosis: 1) Organizational perfusion change; 2) Insufficient body fluids.

Care goals: 1) Maintain the stability of the patient's vital signs; 2) Avoid shock, respiratory cardiac arrest. 3 Patients respond to fluid resuscitation. The patient's blood pressure is shown in Figure 4, and the heart rate is shown in Figure 5.

Care measures: 1) Indwell an indwelling needle in each of the upper limbs. Inject $500 \mathrm{ml}$ of normal saline intravenously according to the doctor's advice; 2 ) After using the warming device for fluid replacement, evaluate the effect of 


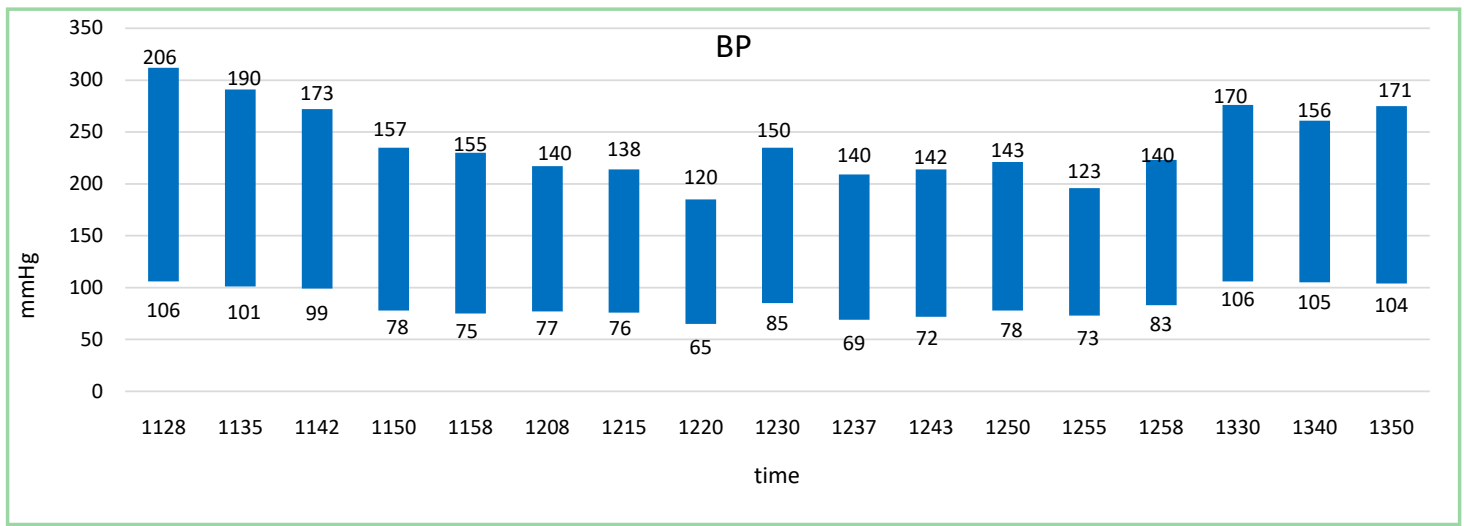

Figure 4. Patient's blood pressure.

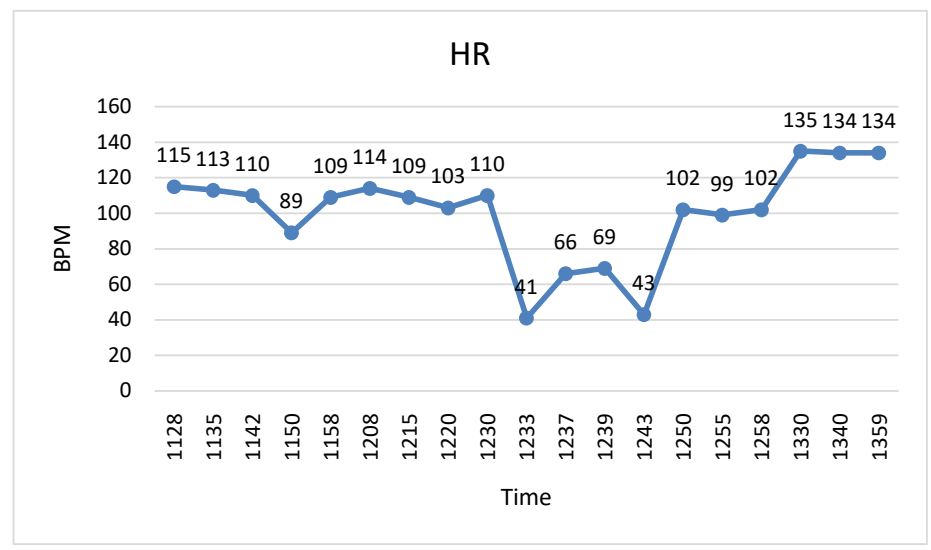

Figure 5. Patient's heart rate.

fluid resuscitation and the patient's response; fluid resuscitation and the patient's response; 3 ) Wrap the head wound to stop the bleeding, and there is no active bleeding in the abrasions; 4) Cooperate with the doctor to perform bedside FAST ultrasound, and the result was negative; 5) Closely observe the bleeding from the wound, protect the fracture site, and immobilize both upper limbs; 6) Observe the changes in the patient's heart rate and blood pressure, and prepare for resuscitation; follow the doctor's instructions to indwell the catheter, export approximately $550 \mathrm{ml}$ of clear urine, and observe and record the urine output.

\subsubsection{Nerve Function}

Nursing assessment: 1) Consciousness: U; 2) GCS: E1V1M1; Right pupil: R 3.0/-, Left pupil: L 3.0/+; 3) Before using sedative drugs and muscle relaxants, the muscle strength of the limbs is 0 ; 4) Perform an axial turn for the patient: no abnormalities in the back, no wounds, intact spine, no bony sensation, no dislocation.

Nursing diagnosis: The ability to regulate intracranial pressure decreases.

Care goals: Avoid secondary damage caused by physical examination or transporting the patient.

Care measures: 1) Closely observe the change of the patient's pupil; 2) Ob- 
serve the patient's blood pressure changes, and if the MAP fluctuation exceeds $50-150 \mathrm{mmHg}$ [9], report to the doctor in time; 3) Observe if there is fluid seeping out of the ear and nose; 4) Before it is determined that the patient's spine and cervical spine are not damaged, continue to fix the spine and wear a cervical collar; 5) During physical examination and operation, try to avoid moving the patient to avoid secondary damage to the patient's fracture site. Notify the CT room.

\subsubsection{Environment and Body Temperature \\ Nursing assessment: $\mathrm{T}: 35.8^{\circ} \mathrm{C}$. \\ Nursing diagnosis: Hypothermia. \\ Care goals: Avoid hypothermia.}

Care measures: 1) Turn off the air conditioning refrigeration of the rescue room and raise the air conditioning temperature; 2) The use of warm cotton from the temperature box is given to the patient to keep warm, to assist the doctor as soon as possible to complete the examination, reduce exposure time; 3 ) Use an infusion warming instrument for infusion; 4) Give patients humane care and protect their privacy.

\subsection{Nursing Evaluation}

The whole rescue process is smooth, the nursing measures are effective, the patient's vital signs are stable when leaving the rescue room, no adverse events occur, and the transfer and evacuation process is safe.

\subsubsection{Risk of Asphyxiation}

15 minutes after the patient entered the rescue room, an artificial high-level airway was established, and airway secretions and blood were cleared in time. No airway obstruction occurred during the rescue.

\subsubsection{Inefficient Breathing Pattern}

Under the coordination of the fiberoptic bronchoscopy, the ordinary tracheal intubation was changed to a double-lumen endotracheal intubation, and after the right lung was sealed and the left lung was used for single-lung ventilation, the patient's blood oxygen rose to over $96 \%$. The Oxygen concentration, patient oxygen saturation and exhalation end carbon dioxide value is shown in Figure 6.

\subsubsection{Tissue Infusion Changes}

Use an infusion warming device for the patient to ensure that the patient is infused with a suitable temperature of liquid, and to avoid the patient's hypothermia after the infusion of a large amount of liquid. During the rescue, we infused the patient with $1500 \mathrm{ml}$ crystalloid. At the same time, indwelling urinary catheterization for the patient, the drained urine is about $550 \mathrm{ml}$. According to the patient's input and output, it can be seen that the patient's renal blood perfusion is acceptable, but it is still necessary to continuously monitor the patient's input and output and make relevant records. The patient's shock index (Shock Index, SI) is less than 1.0, so the patient has no shock correction [9]. Because this patient responded more quickly to fluid resuscitation, the patient lost less blood. 


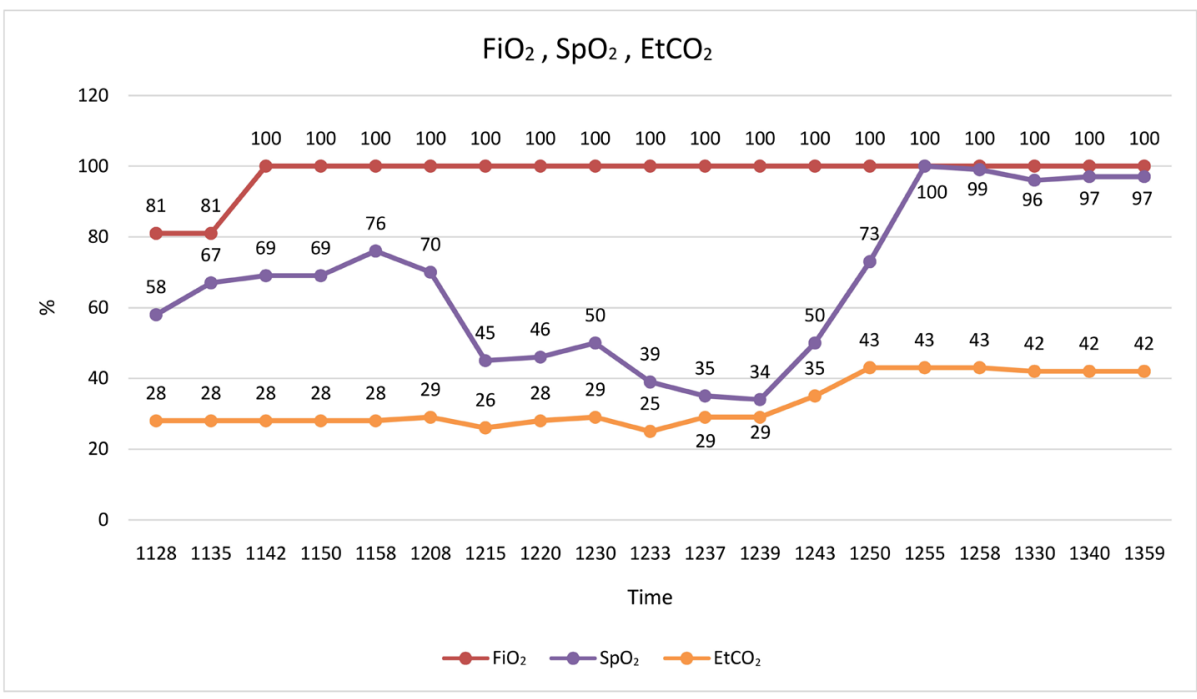

Figure 6. Oxygen concentration and patient oxygen saturation and exhalation end carbon dioxide value.

\subsubsection{Decreased Ability to Regulate Intracranial Pressure}

During the rescue process, the patient was in a deep coma, and no obvious abnormality was found on cranial CT. Due to the use of sedatives, changes in GCS could not be assessed. Although the pupils of the patient changed during the rescue, the $\mathrm{CT}$ scans of the brain after admission were normal. Changes in the pupils of patients are shown in Table 3.

\subsubsection{Hypothermia}

After adopting warming measures and warming the fluid infusion, the patient's body temperature was $35.8^{\circ} \mathrm{C}$ when entering the room, and $36.2^{\circ} \mathrm{C}$ when leaving the room.

\subsubsection{Risk of Respiratory Cardiac Arrest}

After timely chest compressions, the patient quickly resumed spontaneous circulation, and the electrocardiogram showed sinus tachycardia.

\section{Nursing Experience}

In this rescue process, I have real experience: First, for every emergency department nurse, only by correctly understanding trauma care knowledge and proficiently mastering trauma first aid skills, can he be unflappable and at ease in actual clinical work. Second, most nurses can only simply execute medical orders, and lack the critical thinking that is really needed in the clinic. It can be seen that nursing staff need a set of independent nursing procedures in order to correctly assess the patient's condition [10], predict the ever-evolving condition, so as to eliminate the patient's potential hazards in advance, and finally grasp the best time to treat the patient. Therefore, we urgently need a set of simple, effective and systematic nursing procedures to guide patients' rescue procedures in order to improve the survival rate of trauma patients. The ATCN nursing procedure in 
Table 3. Changes in the pupils of patients.

\begin{tabular}{cccc}
\hline Pupil Time & $11: 28-12: 12$ & $12: 15-13: 30$ & $13: 40-13: 49$ \\
\hline Right & $3.0 /-$ & $3.0 /-$ & $4.0 /-$ \\
Left & $3.0 /+$ & $3.0 /-$ & $2.0 /++$ \\
\hline
\end{tabular}

the ATLS guideline is quite different from other traditional nursing procedures. It has the following three advantages: 1) Quick preliminary assessment of trauma patients and taking intervention or treatment measures; 2) It can be dealt with the greatest harm to the life of the patient in the shortest time; 3) In the absence of a definitive clinical diagnosis, taking measures in advance for the potential disease can prevent the occurrence of major potential harm. The ABCDE assessment method and ATCN nursing model in this guideline [11] are not only systematic and efficient, but also can quickly and accurately assess the patient's injury, which has bought us valuable time to treat patients. Therefore, ATLS rescue guidelines and ATCN nursing procedures are worthy of clinical application and promotion.

\subsection{The Important Role of Pre-Hospital Emergency Care in the Treatment of Trauma Patients}

In the first aid process of severe trauma patients, the roles of doctors and nurses are not strictly distinguished, especially in the pre-hospital first aid and nursing process [12]. The seamless connection between pre-hospital and hospital is the key to successful treatment of trauma patients. In the pre-hospital treatment of this case, the medical staff first took active and effective measures to relieve the life-threatening symptoms in a timely manner. An early warning was issued to the hospital, so that the hospital team was fully prepared for treatment before the patient arrived, and at the same time, it also won valuable treatment time for the patient.

\subsection{Implementation of Advanced Trauma Care in Hospitals}

When the patient was first sent to the hospital, the patient's family had not arrived, and we were unable to understand the basic situation of the patient. In this regard, we have opened up a green channel to ensure the smooth progress of rescue work. The first is to use the ABCDE method to conduct a systematic preliminary evaluation of the patient's airway, breathing, circulatory, nervous system, and exposure according to the ATCN process guidelines. At the same time, medical staffs help doctors accurately assess the injury and cooperate with the doctors to rescue them in time, so that they can quickly and accurately judge the patient's injury and relieve life-threatening symptoms in time. Second, after the patient received a series of first aid measures, the gradual stabilization of the breathing cycle provided the doctor with a basic guarantee for further diagnosis and treatment. The third is the cooperation of medical staff to determine the 
priority of the rescue of trauma patients. Fourth, the nurses summed up experience and lessons and put forward constructive opinions through this rescue work. This article summarizes the following points: 1) Nursing staff should learn to use predictive thinking and actively cooperate with doctors to perform diagnostic operations such as FAST ultrasound and bronchoscopy; 2) When performing physical examinations and disease analysis, doctors should do various first aid tasks in advance, such as maintaining the patient's airway unobstructed, establishing venous access as soon as possible, and testing and matching blood in time after blood sampling; 3) Prepare the tracheal intubation according to the patient's injury, and notify the imaging department as soon as possible to perform a bedside chest radiograph; 4) Contact the CT room to make the CT room fully prepared and shorten the inspection time. In summary, the implementation of advanced trauma care measures in the hospital can effectively increase the rescue rate of trauma patients.

\subsection{Pay Attention to Airway Care for Trauma Patients}

For patients with severe trauma, the assessment and management of the airway is the most important step [12]. It is difficult to establish a clear tracheal passage in the early stage. Therefore, physicians and nurses should be familiar with the airway decision-making process of ATLS, and choose an appropriate intubation route to manage the patient's airway [13]. Such patients will suffer from severe hypoxia due to obstruction of airway blood flow, and eventually die from suffocation. In this case, the airway decision-making process of ALTS was used to successfully improve the patient's hypoxia condition through a series of treatment measures.

\subsection{Single Lung Ventilation and Lung Isolation Care}

General departments use single-chamber ventilation, single-chamber isolation, and double-chamber bronchial intubation to improve bronchial obstruction, but it is rarely used in emergency departments. In this case, the anesthesiologist uses a bronchoscope to replace the double-lumen endotracheal tube for single-lung ventilation of the left lung. As shown in Figure 7, the left main bronchus is located at the front end of the catheter and the right main bronchus catheter is balloon-sealed to achieve unilateral ventilation [14]. Finally, in the case of one-lung ventilation, the symptoms of hypoxia are effectively improved. The General Principles guidelines recommend a ventilation volume of $4-6 \mathrm{~mL} / \mathrm{kg}$ (evidence level $2 \mathrm{~B}$ ) to ensure that $\mathrm{ETCO}_{2}$ and $\mathrm{PaCO}_{2}$ are close to the patient's baseline. For patients with normal lung function [15], the guidelines recommend that PEEP be set to $5-10 \mathrm{cmH}_{2} \mathrm{O}$, and in order to maintain a stable blood oxygen concentration, airway hypertension should not exceed $30 \mathrm{cmH}_{2} \mathrm{O}$. Therefore, during the ventilation process, it is necessary to observe whether the thoracic undulation is symmetrical, whether there is subcutaneous emphysema, and the changes of $\mathrm{SpO}_{2}$ and $\mathrm{EtCO}_{2}$. 


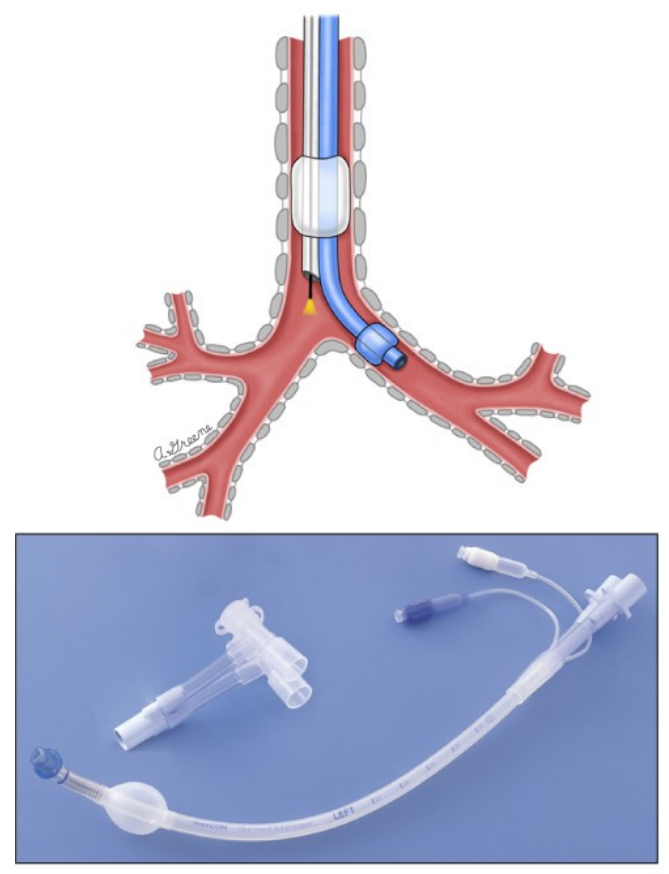

Figure 7. Single lung ventilation model diagram and double cavity trachea intubation.

\subsection{Consider Transshipment as Early as Possible and Give Patients Definitive Treatment}

After the trachea or main bronchus is found to be injured, the patient should be treated immediately [16] [17]. Early surgical treatment can reduce the difficulty and risk of postoperative treatment and promote the rapid recovery of patients. After the patient's vital signs are stable, the patient should be sent to the ward or operating room for further treatment as soon as possible, otherwise the treatment will be delayed and the patient's prognosis will be affected. All in all, when the patient's condition is stable and it is determined that the next surgical intervention is possible, the patient should be transferred to the operating room as soon as possible to shorten the surgical transfer time. In addition, it should be noted that there are many examination and treatment measures for trauma patients [18], so after escorting the patient to the operating room or ward, the SBAR method can be used to compare the patient's examination results, treatment measures, and condition with the operating room nurses. Detailed handover to avoid the omission of important information.

\section{Summary}

Nurses should have a strong sense of first aid and skilled nursing skills to make quick and accurate judgments about emergencies and take first aid measures in time to shorten the rescue time. The training of "trauma nurses" and the introduction of trauma care technology have greatly improved the treatment rate of trauma patients [18]. Traumatology nurses can fully and seamlessly implement comprehensive care for patients, participate in the whole process, rescue and 
transfer safely, reduce nurse handover links, and avoid delays in treatment due to unclear handover [19]. The successful treatment of this case is inseparable from the joint efforts of the medical staff and the optimization of the trauma treatment procedure by the medical staff. Through the theoretical practice of ATLS and ATCN, medical staff can improve the efficiency of treatment, improve the survival rate of patients with severe trauma, and reduce the disability and mortality of patients.

\section{Conflicts of Interest}

The authors declare no conflicts of interest regarding the publication of this paper.

\section{References}

[1] Shemmeri, E. and Vallières, E. (2018) Blunt Tracheobronchial Trauma. Thoracic Surgery Clinics, 28, 429-434. https://doi.org/10.1016/j.thorsurg.2018.04.008

[2] Balci, A.E., Eren, N., Eren, S. and Ulkü, R. (2002) Surgical Treatment of Post-Traumatic Tracheobronchial Injuries: 14-Year Experience. European Journal of Cardio-Thoracic Surgery, 22, 984-989. https://doi.org/10.1016/S1010-7940(02)00591-2

[3] Rice D. C. (2006). Surgery of the Trachea and Bronchi. Annals of Surgery, 244, 158. https://doi.org/10.1097/01.sla.0000226043.56534.fe

[4] Karmy-Jones, R. and Wood, D.E. (2007) Traumatic Injury to the Trachea and Bronchus. Thoracic Surgery Clinics, 17, 35-46. https://doi.org/10.1016/j.thorsurg.2007.03.005

[5] Zhang, Q. and Li, H. (2015) Emergency Nursing Care of a Case of Closed Tracheal Rupture. Northwestern National Defense Medical Journal Chin, 36, 412-413.

[6] Zhou, J. (2018) Trauma Scoring. Science Press, Beijing, 26-30.

[7] Leong, L.M., Chatterjee, S. and Gao, F. (2007) The Effect of Positive End Expiratory Pressure on the Respiratory Profile during One-Lung Ventilation for Thoracotomy. Anaesthesia, 62, 23-26. https://doi.org/10.1111/j.1365-2044.2006.04893.x

[8] Błażewicz, A., Fijałek, Z., Warowna-Grześkiewicz, M., \& Boruta, M. (2007). Simultaneous Determination of Rocuronium and Its Eight Impurities in Pharmaceutical Preparation Using High-Performance Liquid Chromatography with Amperometric Detection. Journal of Chromatography A, 1149, 66-72.

[9] Wu, Z. and Wu, Z. (2008) Surgery. 7th Edition, People's Medical Publishing House, Beijing, 10-12.

[10] Rotondo, M.F. (2012) American College of Surgeons Committee on Trauma. Advanced Trauma Life Support (ATLS) Student Course Manual. 9th Editon, American College of Surgeons, Chicago, 66.

[11] Garvey, P., Liddil, J., Eley, S. and Winfield, S. (2016) Trauma Tactics: Rethinking Trauma Education for Professional Nurses. Journal of Trauma Nursing, 23, 210-214. https://doi.org/10.1097/JTN.0000000000000218

[12] Yang, Q. and Jia, M. (2006) Emergency Rescue of 294 Cases of Trauma. Chinese Journal of Misdiagnosis, 6, 762-763.

[13] Li, X. (2017) Observation and Nursing Countermeasures of Traumatic Main Bronchus Rupture. The Latest Medical Information in the World Abstract, 17, 192-197.

[14] Tao, G., Chen, Q., Feng D., Ge, H. and Liu, W. (2020) Research Progress on the 
Protection of Lung Function in Patients with One-Lung Ventilation. Modern Chinese and Western Medicine Combined Journal, 29, 224-228.

[15] Ma, M. and Peter, D.S. (2020) FRCPC, One Lung Ventilation: General Principles. https://www.uptodate.com/contents/zh-Hans/one-lung-ventilation-general-principl es\#H55334\%201.2020

[16] Mosier, J.M., Joshi, R., Hypes, C., Pacheco, G., Valenzuela, T. and Sakles, J.C. (2015) The Physiologically Difficult Airway. West Journal of Emergency Medicine, 16, 1109-1117. https://doi.org/10.5811/westjem.2015.8.27467

[17] Li, G., Dai, J., Chen, G., et al. (2007) Diagnosis and Treatment of 12 Cases of Traumatic Bronchial Rupture. Chinese Journal of Thoracic and Cardiovascular Surgery Chin, 23, 128 .

[18] Li, S., Li, Y., Liu, H., et al. (2010) Observation on the Effect of Advanced Trauma Nursing in Trauma Emergency Treatment. Nursing Practice and Research, 7, 23-24.

[19] Kaufman, E.J., Richmond, T.S., Wiebe, D.J., Jacoby, S.F. and Holena, D.N. (2017) Patient Experiences of Trauma Resuscitation. JAMA Surgery, 152, 843-850.

https://doi.org/10.1001/jamasurg.2017.1088 